\title{
Protein interactions in the murine cytomegalovirus capsid revealed by cryoEM
}

\author{
Wong H. Hui ${ }^{1,2}$, Qiyi Tang ${ }^{3}$, Hongrong Liu ${ }^{1,2,6}$, Ivo Atanasov ${ }^{1,2}$, Fenyong Liu ${ }^{4}$, Hua Zhu ${ }^{5}$, Z. Hong Zhou ${ }^{1,2 凹 ~}$ \\ ${ }^{1}$ The California NanoSystems Institute (CNSI), University of California, Los Angeles (UCLA), Los Angeles, CA 90095, USA \\ ${ }^{2}$ Department of Microbiology, Immunology and Molecular Genetics, UCLA, Los Angeles, CA 90095, USA \\ ${ }^{3}$ Department of Microbiology/AIDS program, Ponce School of Medicine, 395 Zona Industrial, Reparadara-2, Ponce, PR 00716, \\ USA \\ ${ }^{4}$ School of Public Health, University of California at Berkeley, Berkeley, CA 94720, USA \\ ${ }^{5}$ Department of Microbiology and Molecular Genetics, International Center for Public Health (or ICPH), Room E350D, 225 \\ Warren Street, UMDNJ-New Jersey Medical School, Newark, NJ 07103, USA \\ ${ }^{6}$ Current address: College of Physics and Information Science, Hunan Normal University, Changsha 410081, China \\ $\triangle$ Correspondence: Hong.Zhou@ucla.edu
}

Received July 15, 2013 Accepted August 14, 2013

\begin{abstract}
Cytomegalovirus (CMV) is distinct among members of the Herpesviridae family for having the largest dsDNA genome (230 kb). Packaging of large dsDNA genome is known to give rise to a highly pressurized viral capsid, but molecular interactions conducive to the formation of CMV capsid resistant to pressurization have not been described. Here, we report a cryo electron microscopy (cryoEM) structure of the murine cytomegalovirus (MCMV) capsid at a $9.1 \AA$ resolution and describe the molecular interactions among the $\sim 3000$ protein molecules in the MCMV capsid at the secondary structure level. Secondary structural elements are resolved to provide landmarks for correlating with results from sequence-based prediction and for structure-based homology modeling. The major capsid protein (MCP) upper domain (MCPud) contains $\alpha$-helices and $\beta$-sheets conserved with those in MCPud of herpes simplex virus type 1 (HSV-1), with the largest differences identified as a "saddle loop" region, located at the tip of MCPud and involved in interaction with the smallest capsid protein (SCP). Interactions among the bacteriophage HK97-like floor domain of MCP, the middle domain of MCP, the hook and clamp domains of the triplex proteins (hoop and clamp domains of TRI-1 and clamp domain of TRI-2) contribute to the formation of a mature capsid. These results offer a framework for understanding how cytomegalovirus uses various secondary structural elements of its capsid proteins to build a robust capsid for
\end{abstract}

packaging its large dsDNA genome inside and for attaching unique functional tegument proteins outside.

KEYWORDS cytomegalovirus, herpes simplex virus type 1, cryo electron microscopy, three-dimensional, major capsid protein

\section{INTRODUCTION}

Cytomegalovirus (CMV) infection is a leading viral cause of birth abnormalities. In immuno-suppressed individuals, such as the elderly, patients suffered from AIDS, undergoing organ transplantation, opportunistic CMV infections account for a major portion of life-threatening complications (Britt and Boppana, 2004). Murine CMV (MCMV) infections of mice are a commonly used disease model for studying human CMV (HCMV) infections. As a prototype of the beta-herpesvirus subfamily of the Herpesviridae family, CMV has a dsDNA genome of $230 \mathrm{~kb}$, the largest among all herpesviruses. The large genome encodes over 200 proteins, including both proteins that are conserved among other herpesviruses and several unique proteins that lack homologs in alpha- or gamma-herpesviruses (Mocarski, 2005). The DNA genome is packed inside an icosahedral proteinaceous capsid, which is surrounded by layers of tegument proteins, which, in turn, is enclosed within a lipid envelope decorated with trans-membrane glycoproteins. During infection, the DNA-containing capsid is first released in the host cytoplasm and then transported to the nuclear pore complex, through which the DNA is ejected into the host nucleus. Therefore, the capsid plays important roles throughout the life cycle of CMV infection to ensure the encapsidation and deliv- 
ery of viral DNA.

Previous cryo electron microscopy (cryoEM) studies have determined molecular resolution three-dimensional (3D) structures of CMV capsids (Butcher et al., 1998; Chen et al., 1999; Trus et al., 1999; Yu et al., 2005a). Together with previous biochemical studies, these studies have established that the CMV capsid has a single-shelled $\mathrm{T}=16$ icosahedral capsid composed of four major proteins: the major capsid protein (MCP; encoded by UL86 in HCMV and M86 in MCMV) (Chee et al., 1989), the minor capsid protein ( $\mathrm{mCP}$ or TRI-1; encoded by UL85 in HCMV and M85 in MCMV), the MCP binding protein (mC-BP or TRI-2; encoded by UL46 in HCMV and M46 in MCMV) (Gibson et al., 1996a), and the smallest capsid protein (SCP; encoded by UL48.5 in HCMV and M48.5 in MCMV) (Baldick and Shenk, 1996; Gibson et al., 1996b; Borst et al., 2001). As shown by structural studies of other herpesviruses (e.g., Booy et al., 1991; Wu et al., 2000; Zhou et al., 2000; Trus et al., 2001; Yu et al., 2003), the CMV capsid shell has a structure similar to those of herpes simplex virus type 1 (HSV-1) (e.g., Booy et al., 1991; Zhou et al., 1994, 1995, 2000) and Kaposi's sarcoma-associated herpesvirus (KSHV) (Wu et al., 2000; Trus et al., 2001). By analogy to the capsid architectures of and protein assignments in other herpesviruses, these proteins are organized on a $T=16$ icosahedral capsid shell with pentons and hexons capsomers (pentomer and hexamers of MCP and SCP) and triplex heterotrimers (one TRI-1 and two TRI-2 molecules) connecting the capsomers (Reviewed by Liu and Zhou, 2007).

However, the structures of CMV capsid are known only to molecular resolution. In the absence of structure information of CMV capsid proteins at the levels of molecular domains and secondary structural elements, questions essential to understanding capsid assembly and stability remain largely unanswered. For example, it remains unknown how domains of MCP subunits associate to form capsomers, how domains of TRI-1 and TRI-2 interact to form triplex heterotrimers, and how capsomers and triplex heterotrimers interact with one another to form a highly stable CMV capsid to withhold large internal pressure exerted by the large dsDNA genome.

In this paper, we report the $3 \mathrm{D}$ structure of MCMV capsid about $9.1 \AA$ resolution by single particle cryoEM. Secondary structural elements resolved in the cryoEM structure were combined with sequence-based structure prediction and homology modeling to identify molecular interactions important for the formation of a highly stable capsid against pressurization. Structural differences from the capsids of alpha-herpesvirus and gamma-herpesvirus are discussed in the contexts of differences in association with SCP and tegument proteins.

\section{RESULTS}

\section{Overall structure of MCMV capsid}

As detailed in the Materials and Methods section, we first processed $\sim 10,000$ MCMV capsid images boxed out from the Polara CCD micrographs. The resolution of the $3 \mathrm{D}$ reconstruction from these Polara micrographs converged at about $11 \AA$ and further effort to sort out 'good' particle images based on particle quality did not improve the resolution, suggesting limited data quality of these Polara micrographs. To further improve resolution, we subsequently recorded a new data set of the same MCMV capsid sample on photographic films in a Titan Krios instrument operated at $300 \mathrm{kV}$. By averaging 3467 capsid images (Fig. $1 \mathrm{~A}$ ), we obtained a $3 \mathrm{D}$ reconstruction of the MCMV capsid at a resolution of $9.1 \AA$ (Fig. 1B-D, Fig. S1 and Movie S1). Typical of cryoEM structures at subnanometer resolution, our MCMV capsid map reveals rod-like and sheet-shaped densities, corresponding to $\alpha$-helices and $\beta$-sheets, respectively. The visualization and identification of secondary structure elements in the capsid proteins allowed us to describe molecular interactions among the three capsid proteins of a cytomegalovirus capsid for the first time.

The 1310 A-diameter, icosahedrally averaged MCMV capsid has the characteristic $T=16$ icosahedral symmetry with 12 pentons, 150 hexons and 320 triplexes (Fig. 1B-D). The structural components within an asymmetric unit include one subunit of a penton ("5" in Fig. 1B), 2.5 hexons (one P hexon, one $C$ hexon and $1 / 2 \mathrm{E}$ hexons), and five and $1 / 3$ triplexes ( $\mathrm{Ta}$, Tb, Tc, Td, Te, and 1/3 Tf) (Fig. 1C). Penton and hexon are both cylindrically shaped each with a central channel passing through the cylinder and contain five and six subunits, respectively. Triplex Ta, Tb, Tc, Td triplexes are located on the local 3-fold axes and triplex Tf is on the icosahedral 3-fold axis. Each triplex connects either three hexons or a penton and two hexons.

\section{Upper domain of MCP}

MCP (1353 aa) is the constituent of both penton and hexon subunits, totaling 960 copies per capsid and accounting for $3 / 4$ of the total mass of the capsid shell. At $9.1 \AA$ resolution, the bulk of the molecular boundaries are resolved (Fig. $1 \mathrm{C}$ and $1 D$ ), allowing for the segmentation of a single subunit from the averaged hexon (Fig. 2A). In the capsids of CMV and other herpesviruses, SCP is known to bind MCP upper domain. A previous cryoEM reconstruction of anti-SCP antibody labeled CMV capsids has localized SCP bound to the outermost region of MCP subunits in hexons (Yu et al., 2005a) and boundary between SCP and MCP can be estimated by varying the density threshold and by fitting with atomic models of MCPud (Figs. $2 \mathrm{~A}, 2 \mathrm{~B}, 3 \mathrm{~A}$ and $3 \mathrm{~B}$ ). In our $9.1 \AA$ map, a density similar to that of the SCP density in hexon is also located in the outmost region of penton MCP. The fact that anti-SCP failed to recognize the penton SCP (Yu et al., 2005a) suggests different antigenicity of the penton and hexon SCP molecules, perhaps mirroring the different antigenicity of penton and hexon MCP subunits previously observed (Trus et al., 1992). Our map shows that each SCP has three helices, the longest of which interacts with a horizontal helix in the upper domain of MCP (Fig. 2A).

To facilitate description of structural arrangement and molecular interactions, we divide each MCP subunit into at least four domains: an upper domain, a middle domain, a dimerization domain and a floor domain (Fig. 2A). These four domains 

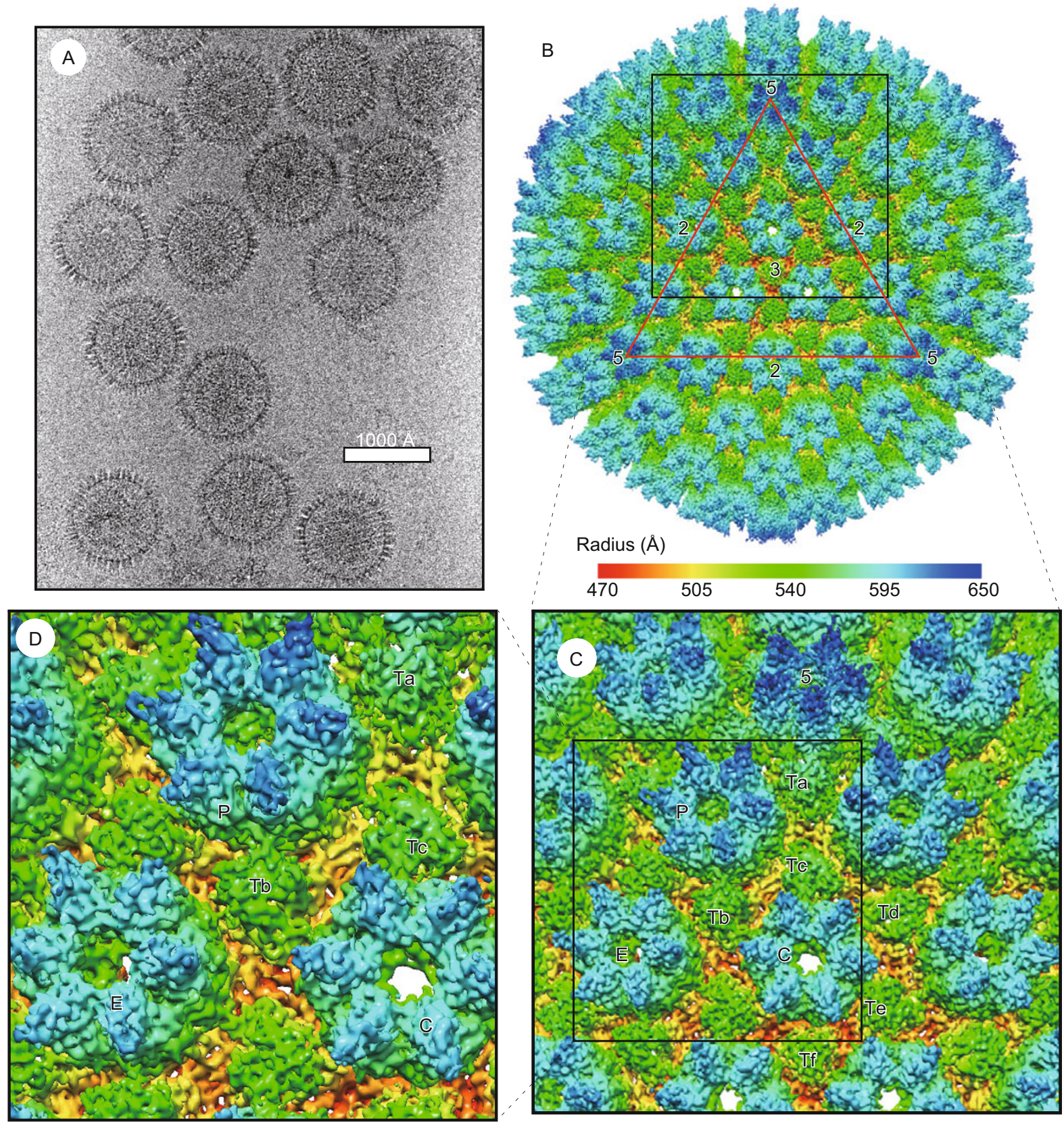

Figure 1. CryoEM and 3D reconstruction of MCMV capsid. (A) Representative raw cryoEM micrograph of MCMV capsids recorded in a Titan Krios instrument operated at $300 \mathrm{kV}$. (B) Shaded surface representation of the MCMV capsid reconstruction at $\sim 9.1 \AA$ resolution colored radially according to the color bar shown below. (C-D) Two consecutive close-up views of the MCMV capsid reconstruction. Structural components in an asymmetric unit are labeled, including penton (“5”), C hexon ("P"), E hexon (“E”), P hexon ("P"), and six types of triplex heterotrimers Ta, Tb, Tc, Td, Te, Tf.

all contain a mixture of $\alpha$-helices and $\beta$-sheets, thus having the $\alpha+\beta$ structure type. The current resolution of the map does not permit directly tracing the polypeptide chain based on our cryoEM density map alone. Nonetheless, for the upper and floor domains, atomic models of structurally homologous proteins are available from $\mathrm{X}$-ray crystallography and were used to map some amino acid sequence segments to the second- ary structures observed in our cryoEM densities.

Structural interpretation of the MCMV MCP upper domain is aided by the crystal structure of the MCP upper domain (MCPud, i.e., MCP aa 484-1045) of HSV-1 (Bowman et al., 2003). The sequence identity between the upper domains of MCMV and HSV-1 MCP is $21 \%$ (Fig. S2). Fitting of the HSV-1 MCPud crystal structure into our cryoEM density map 


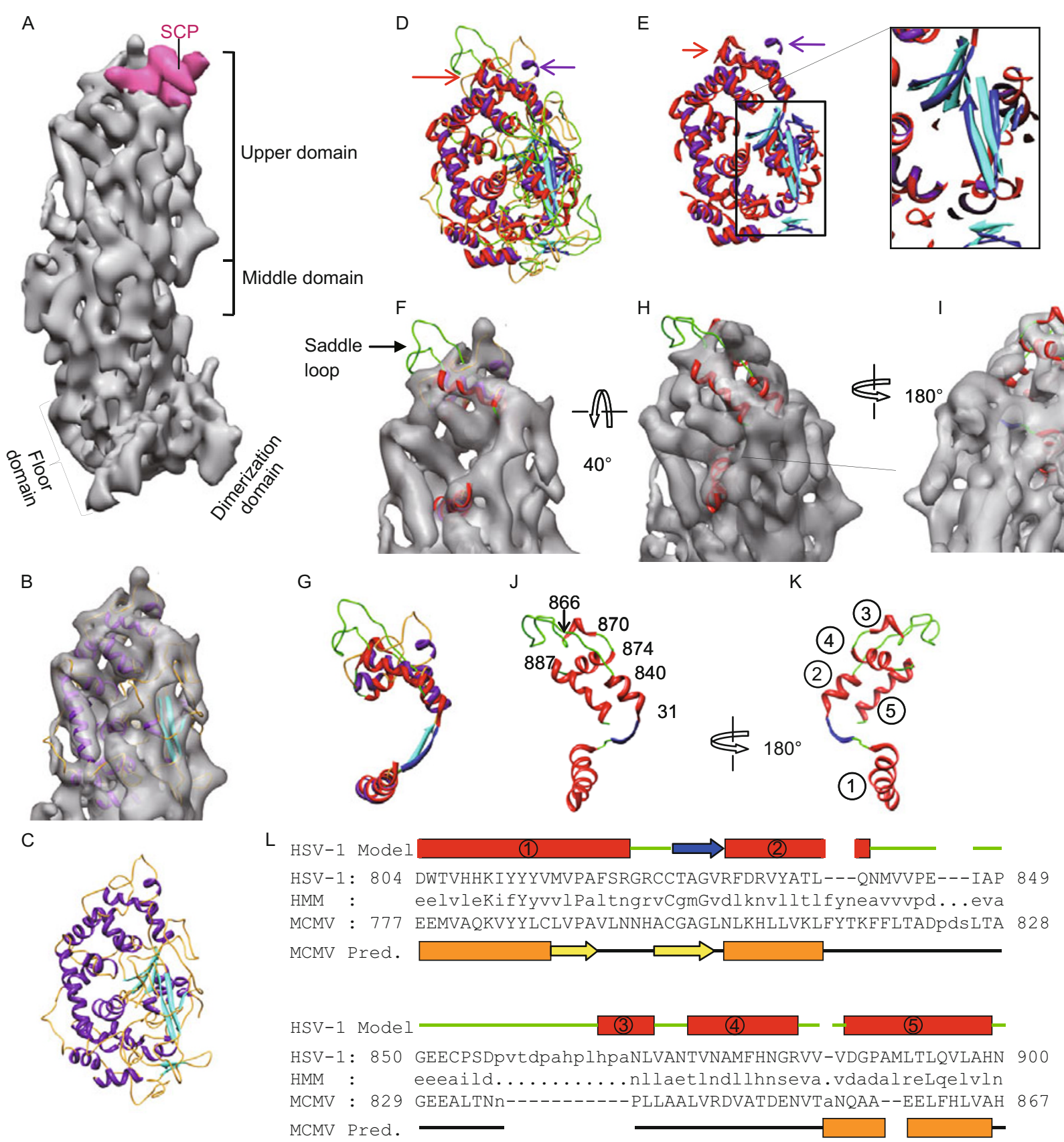

Figure 2. MCMV MCP cryoEM structure and comparison with HSV-1 MCPud. (A) Side view of a subunit segmented from the C hexon. Each hexon subunit contains one molecule of MCP (gray and its domains labeled) and one molecule of SCP (pink). (B) CryoEM density map (semi-transparent gray) of the MCP upper domain (MCPud) superimposed with its pseudo-atomic model (ribbon). The pseudo-atomic model was built using the X-ray model of the HSV-1 MCPud as template and the MCMV cryoEM structure as constraints (see Materials and Methods). (C) Only show the pseudo-atomic model from (B). (D) Comparison of HSV-1 MCPud X-ray model with the pseudo-atomic model for MCMV MCPud. (E) Close-up view of (D), however loops of both models are removed. (F) Comparison of MCMV MCPud cryoEM density map with atomic structure of HSV-1 MCP residues from 802-900. It focuses on structure difference of the saddle loops. (G) Comparison of atomic structure of HSV-1 MCP residues from 802-900 with corresponding part of pseudo-atomic model of MCMV MCPud. $(\mathrm{H}, \mathrm{I})$ Front $(\mathrm{H})$ and back $(\mathrm{I})$ views of MCMV MCPud superimposed with the crystal structure of HSV-1 MCP residues from 802-900. (J, K) Same as that of $(\mathrm{H}, \mathrm{I})$ but the MCMV structure are removed and residue numbers $(\mathrm{J})$ and helices are labeled $(\mathrm{K})$. (L) Alignment between the MCP molecules of MCMV with HSV-1, obtained from the Pfam Sequence Search server (http://pfam.sanger. ac.uk/search). Only the result corresponding to residues 804-900 of HSV-1 MCP are shown. The secondary structure model for HSV-1 MCP is based on the crystal structure feature (Bowman et al., 2003) and that for the MCMV MCP is based on Jpred 3 secondary structure prediction server (http://www.compbio.dundee.ac.uk/www-jpred/). Rectangles stand for helices, arrows stand for strands and strings for loops. HMM: Hidden Markov Model depicting the consensus sequences among the major capsid proteins of many herpesviruses identified by the Pfam Server. 
of MCMV MCPud shows that all helices with three or more turns and all four $\beta$-sheets in HSV-1 MCPud have positional counterparts in our cryoEM density map of MCMV MCP (Movie S2). Using the secondary structure elements identified in the MCMV MCP structure as constraints, we obtained a homology model for the MCMV MCPud using the HSV-1 MCPud as a template (Fig. 2B and 2C).

Superposition of the MCMV MCPud homology model and the HSV-1 MCPud X-ray model shows that the most significant structural difference between MCMV MCPud and HSV-1 MCPud is located at their outmost regions (Fig. 2D and Movie S2). In the crystal structure of HSV-1 MCPud, this region contains aa $841-865$ as loops folded into the shape of a horse saddle (green in Fig. 2F-H). In the crystal structure of HSV-1 MCPud, this saddle structure is flanked by a 2.5-turn $\alpha$-helix (helix 2: aa 831-840) to its N-terminal side and by two short helices (helix 3: aa 866-870 and helix 4: aa 874-883) to its Cterminal side (Fig. $2 \mathrm{~J}$ and $2 \mathrm{~K}$ ). In the MCMV cryoEM structure, the density corresponding to the saddle structure is shifted by $\sim 15 \AA$ away from the hexon channel axis along the helix 5 . As a result, the HSV-1 MCPud saddle structure does not fit in the cryoEM map of MCMV (Fig. 2D-L and Movie S2). The three short helices (helices 2, 3, 4) flanking the saddle also exhibit large structural differences between MCMV and HSV-1 MCPud. Other helices (helix 1 and 5) are almost identical between the MCMV MCP cryoEM structure and the HSV-1 MCP crystal structure (Fig. 2G-K).

In our homology model of MCMV MCPud, the saddle loop structure corresponds to aa 777-867 of MCMV MCPud (Figs. 2B, 2C, 2G, 2L and S2). Compared to HSV-1 MCP, this region of the MCMV MCPud has two insertions 3 residues each (MCMV MCP aa 813-815 and aa 823-825) and a long deletion of 11 residues (HSV MCP aa 858-868) (Fig. 2L). These insertions and deletion are consistent with the large differences between the saddle structures of HSV-1 and MCMV.

\section{Floor domain of MCP and the HK97-like fold}

We examine the role of the MCP floor domain by inspecting the interactions among the floor domains within a hexon (Fig. 3A-C). The top view of the floor domains in the hexon shows that six large $\beta$-sheets, one in each MCP monomer, contact and form a ring (Fig. 3C). Each $\beta$-sheet consists of two consecutive $\beta$-sheets connected by small density (likely a loop) (Fig. $3 \mathrm{H}$ and $3 \mathrm{~K}$ ). It has been suggested that the floor domain of HSV-1 MCP contains a highly conserved bacteriophage HK97-like canonical ('Johnson') fold (Baker et al., 2005).

To examine how this fold is conserved in MCMV, we fit the crystal structure of bacteriophage HK97 gp5 (Wikoff et al., 2000) in the floor domain of MCMV MCP. The gp5 structure fits well with the floor domain, confirming the similarities of their overall folds (Fig. 3G and 3H), including four $\beta$-sheets (Fig. 3G-L).

The fitting also revealed structural differences between the HK97 gp5 and the floor domain of MCMV MCP. HK97 gp5 has 3 long helices (Fig. 3E). The longest helix ("spine" helix) labeled as helix 1 and the third longest helix labeled as helix 3 are well conserved, but one end of the second longest helix tilts away about $5 \AA$ from the corresponding helix in the density map (Fig. 3G-J). The longest helix of MCMV is $58 \AA$ and is longer than its corresponding long spine helix in HK97 gp5. Secondary structure prediction (Fig. S3) indicates that the longest helix is also about $58 \AA$ in length and is close to the N-terminal of MCP (aa 148-187). Proceeding to this spine helix are a short helix and a set of $\beta$-strands, similar as those in $\mathrm{N}$-terminal of HK97 phage. This assignment of $\mathrm{N}$-terminal segment of MCP to the floor domain is consistent with mutagenesis data demonstrating $\mathrm{N}$-terminal sequence interacting with internal scaffolding protein (Huang et al., 2007). The spine helix in the cryoEM structure is sandwiched between the above mentioned consecutive $\beta$-sheets and a long loop (Fig. $3 \mathrm{H}$ ).

\section{Triplex heterotrimer}

Each triplex heterotrimer consists of one monomer of TRI-1 and two monomers of TRI-2. The cryoEM structures of all six triplexes have the shape of a truncated pyramid with a triangular top (left panels in Fig. 4A-C and Movie S3). Except for Tf, which is on the icosahedral 3-fold axis, the other triplexes are asymmetric. Ta differs slightly from the rest of asymmetric triplexes (Tb, Tc, Td and Te), likely due to its location near the vertex.

We first identify the TRI-1 monomer and TRI-2 dimer in the triplex heterotrimer in our cryoEM structure by correlating secondary structural elements observed in our cryoEM density map and those predicted from the M46 and M85 sequences. In HSV-1, it has been shown that each triplex consists of a monomer (VP19c) and a dimmer (VP23), which are the homologs of the M46-encoded minor capsid protein and the M85-encoded minor capsid binding protein of MCMV, respectively. We segmented out the three subunits in each triplex of the MCMV capsid by careful examination of the secondary structure and their connectivity identifies within the averaged density map of the triplex (Fig. 4A). Two of the three subunits within each triplex are similar to one another (the yellow and green subunits in Fig. 4A and Movie S3). In particular, both of these subunits contain three long (>20 $\AA$ in length) helices and several short helices (green and yellow in 2nd and 3rd columns of Fig. 4B and $4 \mathrm{C}$ ) and are thus assigned to be the TRI-2 dimer. The third subunit (purple subunit in Fig. 4A), now assigned to TRI-1 by way of elimination, has a $\beta$-sheet-rich body with a helix-rich hook domain and a loop-like hand (Fig. 4A and 4D). The characteristics of the observed secondary structure contents are consistent with the predicted secondary structure elements of both TRI-1 and TRI-2 based on their sequences (Figs. S4 and S5).

To facilitate description of structural arrangement and molecular interactions, we divide each TRI-2 monomer into three domains from bottom to top as: clamp, trunk and embracing arm. Six helices are clearly visible in each TRI-2 and are labeled as helix 1-6 (the right three columns in Fig. 4B and 4C). Helices 3 through 6 form the embracing arm of TRI-2. The two 

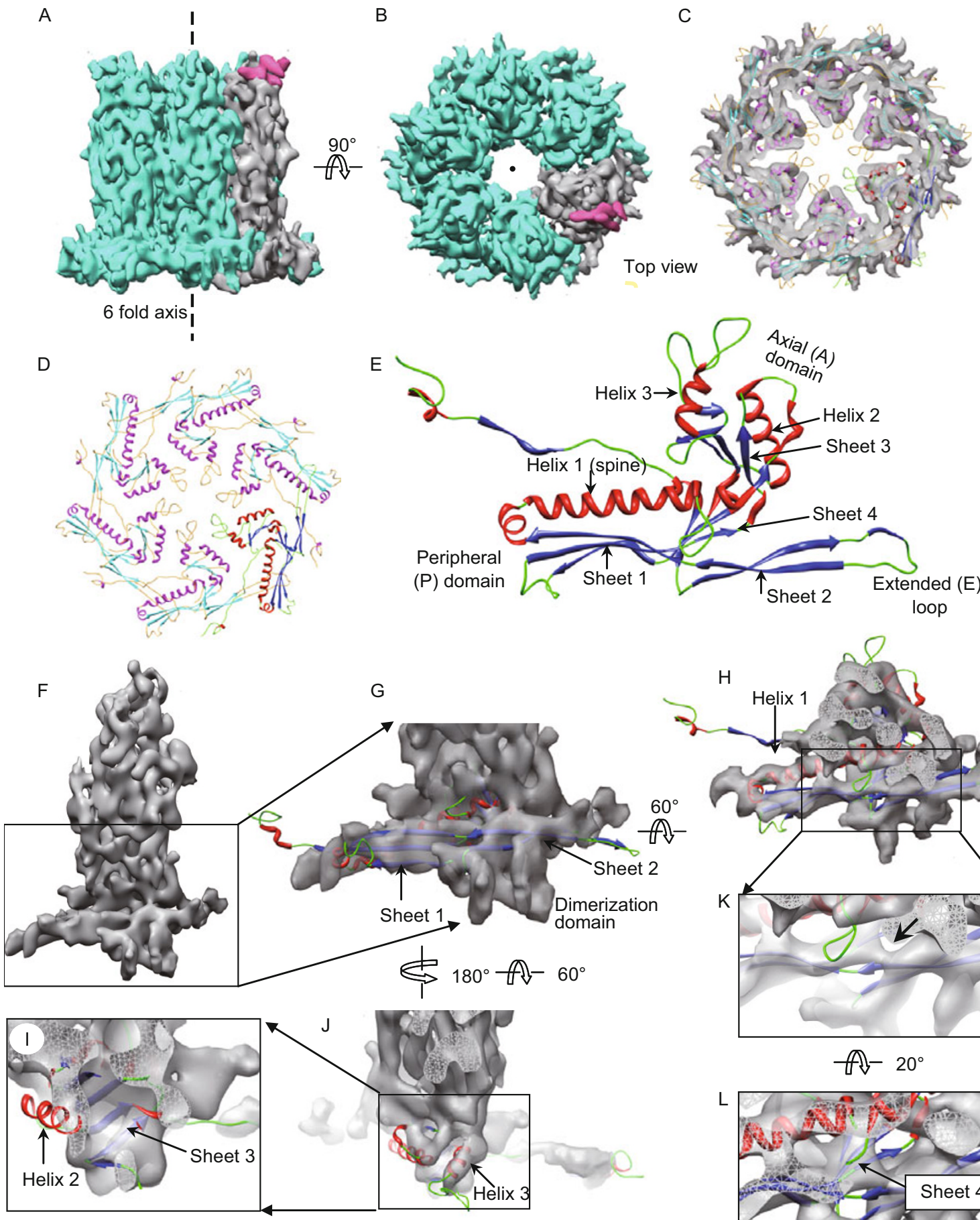

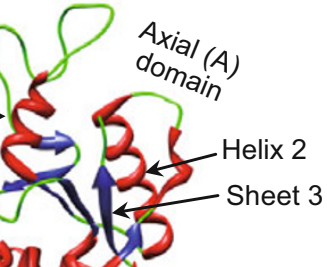

\section{sis}
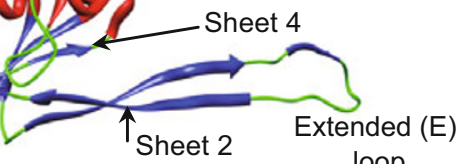

loop

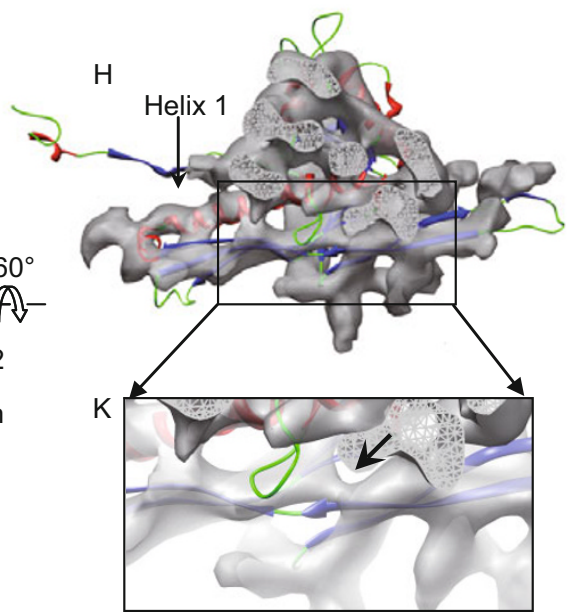

तु- $20^{\circ}$

L

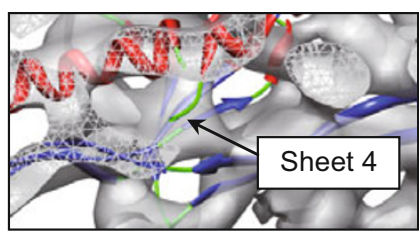

Figure 3. MCP structure and structural organization of MCP floor domain. (A and B) Side (A) and top (B) views of C hexon. (C) Same as (B) but its top $95 \AA$ has been removed to show a density slab of the floor region, superimposed with six copies of the HK97 gp5 crystal structures (ribbons). (D) Same as in (C) but the cryoEM density map has been removed to show the six molecules of the HK97 gp5. (E) Crystal structure of HK97 gp5 monomer. (F-L) C hexon subunit (F) and consecutive close-up views (G-L) of its floor domain with gp5 crystal structure (ribbons) docked in.

TRI-2 monomers within each triplex embrace each other by crisscrossing a pair of long helices (helix 3 and 6 ) and by holding together with two short helices (helix 4 and 5) (Fig. 4B and
4C). Helix 3 and 6 in TRI-2b (yellow) run roughly parallel but helix 6 is tilted by $\sim 30^{\circ}$ in TRI-2a (green one) (Fig. 4B and $4 \mathrm{C}$ ).

Three interactions can be identified between TRI-1 and TRI-2 

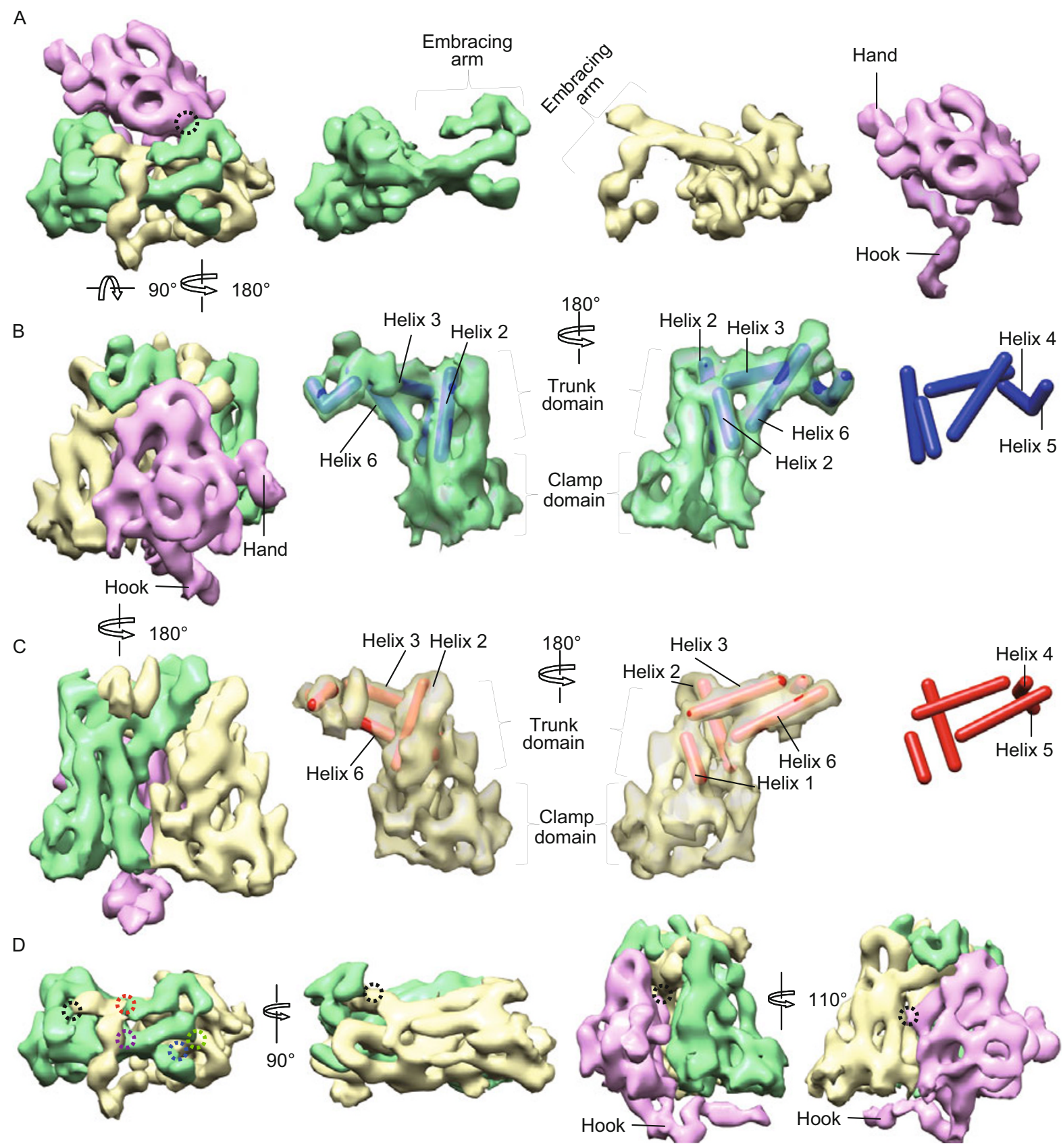

Figure 4. Structure of the molecular clamp-triplex heterotrimer. $(A-C)$ Top $(A)$ and two side $(B$ and $C$ ) views of the triplex heterotrimer. The average triplex structure was obtained by averaging triplexes Tb, Tc, Td, Te. The TRI-1 subunit (purple) and two TRI-2 conformers are segmented and shown in different colors (TRI-2a in green, TRI-2b in yellow, and TRI-1 in magenta). In the second, third and fourth columns of $(A)$, the three triplex monomers are shown alone. In the two middle panels of $(B$ and $C)$, the TRI-2 conformer subunits are shown semi-transparently and superimposed with their helices (cylinders). In the right panel of (B, C), only the helices are shown. (D) Interactions between TRI-2a and TRI-2b and between TRI-2 conformers and TRI-1 monomer are indicated by dotted circles.

(Fig. 4A and 4D). First, the top of TRI-1 connects with the helix 5 connecting with above arm of TRI-2a (Fig. 4A, highlighted by the dotted circle on the first column). Second, the trunk domain of TRI-1 attaches to the helix 6 of TRI-2b underneath (Fig. 4D, highlighted by the dotted circle on 3rd column). Third, a protruding area of TRI-1 connects to the top of helix 3 of TRI-2b
(Fig. 4D, highlighted by the dotted circle on 4th column).

Interestingly, secondary structure prediction of the TRI-1 homologs from three herpesvirus sub-families suggests that beta-herpesvirus has a structure (aa 46-293 of MCMV TRI-1) similar to TRl-1 of gamma-herpesvirus but different from that of alpha-herpesvirus (Fig S5). TRI-1 of HSV-1 has an insertion 
of 49 residues near the C-terminal region, consistent with its larger structure (Zhou et al., 2000).

\section{Interaction between MCP and triplex heterotrimer}

Each triplex heterotrimer sits on the floor domains of three surrounding capsomers (i.e., three hexons, or two hexons plus one penton) (Fig. 5A and Movie S4), totaling 320 triplexes in each capsid. As a whole, each triplex heterotrimer acts as a molecular clamp by interacting with six surrounding MCP monomers in the three neighboring capsomers. There are three kinds of interactions between triplex and surrounding MCPs: (1) sides of triplex and middle domains of MCPs; (2) bottom of triplex with the floor domains of MCPs from outside; (3) hook of TRI-1 with floor domains of MCPs from inside.

The small hand-like density protruding out from the body of TRI-1 (left panel in Fig. 4B) interacts with the middle domain of its neighboring MCP (Fig. 5A-D). The U-shaped density containing helix 5 of TRI-2b (the yellow monomer in Fig. $5 \mathrm{~A}$ and $5 \mathrm{~B}$ ) also interact with the middle domain of its neighboring $\mathrm{MCP}$ (Fig. 5B, 5E and 5F). In contrast, no such interactions are observed between TRI-2a and its neighboring MCP (Fig. 5B), suggesting conformational difference in the corresponding region of TRI-2a.

The most salient observation is that an extended, helix-rich hook domain of TRI-1 traverse the capsid shell to reach the inside of the capsid (Fig. 4D) and anchors the entire triplex complex from underneath the $\beta$-sheets of the MCP floor domains of two MCPs (Fig. $5 \mathrm{G}$ and $5 \mathrm{H}$ ). This anchoring interaction may function to drive tightening adjacent MCP floor domains during transition from the spherical, procapsid to angular, robust capsid common among all herpesviruses (Heymann et al., 2003; Yu et al., 2005b; Deng et al., 2008).

\section{DISCUSSION}

The structure of MCMV capsid offers some general insight into the architectural designs of herpesvirus capsid. Covalent protein chainmail formed by the Johnson fold was first observed in bacteriophage HK97 capsid protein gp5 for forming a stable capsid capable to resist high internal pressure (Wikoff et al., 2000). Though lacking recognizable sequence homology, the structural similarity between the floor domain of MCMV MCP and HK97 gp5 is striking — both containing a Peripheral domain containing a $\beta$-sheet and a long ('spine') helix, an axial domain for capsomer formation, an extended loop and a flexible $\mathrm{N}$-arm. This striking similarity reinforces the notion that dsDNA viruses use the Johnson fold to build capsids to withhold the enormous internal pressure exerted by their dsDNA genomes.

However, there are marked differences between MCMV and HK97 in the overall structures of MCMV MCP and HK97 gp5. HK97 ( $T=7$ and $660 \AA$ in diameter) uses only one protein, gp5, that is one-quarter the size of MCP of CMV and comprised of nothing more than the original Johnson fold to build its capsid. Cytomegalovirus capsid ( $T=16$ and $1310 \AA$ in diameter) is much more structurally complex and larger, and uses a much larger MCP and two more proteins, TRI-1 and TRI-2, to construct its capsid. Inside the Johnson fold, cytomegalovirus MCP has a dimerization domain for joining two hexons or a hexon with a penton. Outside the conserved Johnson fold floor domain, cytomegalovirus MCP is adapted to accommodate prominent domain insertion (totally $\sim 3$ times the full size of the HK97 gp5) at the corresponding axial domain location in HK97 gp5. The insertion gives rise to the protruding middle and upper domains of the capsomer, characteristic of all herpesvirus capsids (Fig. 3E-G). These inserted domains mediate packaging of functionally-important, but asymmetrically-organized outer components of the herpesviruses, including tegument proteins specific to beta-herpesviruses (see below).

The structural features that most clearly differentiate capsids of beta-herpesvirus and alpha-herpesviruses (e.g., HSV-1) are located at the distal ends of MCP (Fig. 2D, 2F and 2G). Our MCMV capsid structure reveals that the structure of betaherpesvirus MCPud is similar to that of the HSV-1 MCPud, up to the level of secondary structures with most $\alpha$-helices and $\beta$-sheets of HSV-1 MCPud are conserved in MCMV MCPud. However, at the top of HSV-1 MCPud there is a saddle loop which is connecting to one small helix at the $\mathrm{N}$-terminal side and two small helices at the C-terminal side. Comparing to the X-ray structure of HSV-1 MCPud, this saddle loop has a different conformation, and the position of this saddle loop, together with its three adjacent helices of MCMCV MCPud, is located farther away from the hexon central channel (Movie S2).

These distal regions of MCP in HSV-1 and CMV are involved in the association of SCP and proteins in the inner tegument layer (Chen et al., 1999; Trus et al., 1999; Zhou et al., 1999) and the observed structural difference in this region is consistent with the diversities of SCP and inner tegument proteins across sub-families of herpesviruses. SCP is the least conserved capsid protein among viruses across subfamilies of herpesviruses, varying in size the greatest among all capsid proteins (75 aa in CMV, 112 aa in alpha-herpesviruses and 170 aa in gamma-herpesviruses). The patterns of capsid-tegument protein interactions also vary greatly across different subfamilies of herpesviruses (Chen et al., 1999; Trus et al., 1999; Zhou et al., 1999). In CMV, the inner tegument layer consists of highly organized filamentous proteins interacting with the pentons, hexons and triplexes of the capsid (Chen et al., 1999; Trus et al., 1999; Mettenleiter, 2002). Whereas in alpha-herpesviruses, tegument proteins bind only to pentons and its 10 surrounding triplexes (Zhou et al., 1999; Liu and Zhou, 2007; Trus et al., 2007; Conway et al., 2010; Toropova et al., 2011; Homa et al., 2013). Thus, the observed structural difference in the MCP upper domain (Fig. 2 and Movie S2) across alpha-, beta- and gamma-herpesviruses correlates well with the differences in the association of these capsids with overlying tegument proteins and may be biologically relevant to different herpesvirus infections.

The upper domains of triplex proteins in alpha-herpesvirus and beta-herpesvirus also bind different tegument densities, a defining feature of specific capsid-tegument associations 


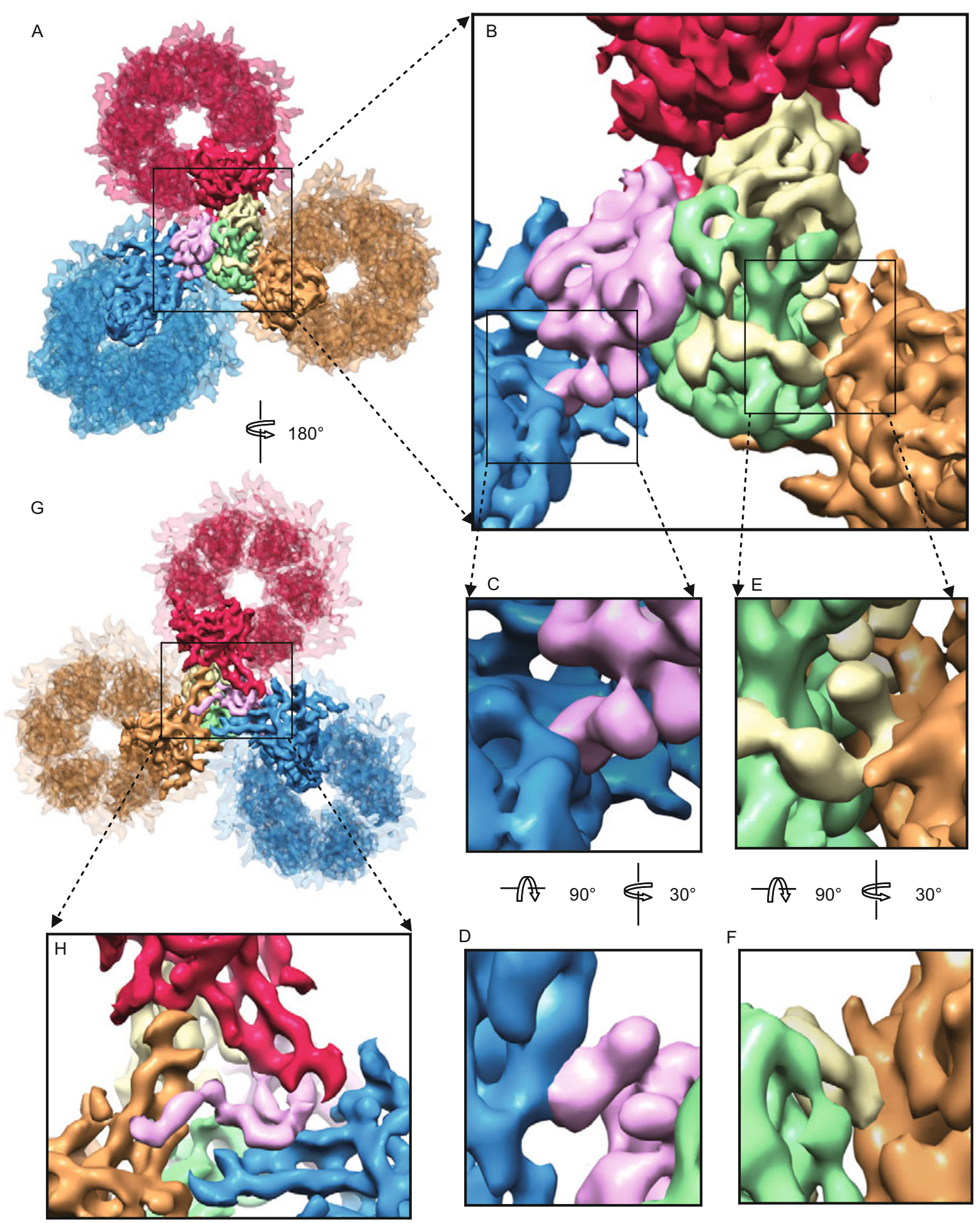

Figure 5. Interactions between triplex and MCP. (A) Top view of triplex with its three surrounded hexons. (B-F) Consecutive close-up (B, $C$ and $E$ ) and side $(D$ and $F$ ) views of $(A)$ showing the interaction between MCP with triplex monomers TRI-1 $(C$ and $D)$ and with TRI-2b (E and $F)$. (G) Back view of $(A)$. (H) Close-up view of $(G)$.

across different herpesvirus subfamilies (Chen et al., 1999; Zhou et al., 1999; Liu and Zhou, 2007; Trus et al., 2007; Conway et al., 2010; Toropova et al., 2011; Homa et al., 2013).
The upper domain of TRI-1 is highly divergent across different herpesvirus subfamilies; for instance, the structure of the HSV-1 TRI-1 homolog, VP19c, is substantially larger, consistent with 
its $\sim 30 \%$ longer sequence than that of CMV TRI-1 (Zhou et al., 2000; Okoye et al., 2006). These differences are consistent with the divergent roles of the triplex proteins in mediating the attachment of divergent tegument proteins in different subfamilies of herpesviruses (Chen et al., 1999; Zhou et al., 1999; Liu and Zhou, 2007).

Among the three structural layers (capsid, tegument and envelope) that are common among all herpesviruses, the tegument layer is the most divergent and contains components that are critical to establishing lytic infection and latency in specific host cell types, as well as to maintaining capsid integrity during DNA encapsidation to generate progeny viral particles. It follows that these viruses must have evolved to possess divergent sets of tegument proteins and regions of the capsid proteins interacting with these divergent tegument proteins have to adapt accordingly. The secondary structure elements resolved in the current study provide direct evidences supporting this point of view.

In summary, the first subnanometer-resolution structure of a beta-herpesvirus capsid reported here reveals bacteriophage HK97-like fold in the capsid floor domain of the major capsid protein. Through large domain insertion to this highly conserved fold, and addition of the clamping triplex heterotrimers, the cytomegalovirus capsid has evolved into a substantially more complex structure as compared to the bacteriophage HK97. Even for viruses within the Herpesviridae, evolutionary pressures towards achieving optimal fitness within their specific host cells have pushed them to also adopt marked structural differences in the outer portions of their capsids to accommodate overlying, functional tegument proteins which are highly divergent from viruses across different subfamilies.

\section{MATERIALS AND METHODS}

Virus preparation

MCMV strain Smith was purchased from American Type Tissue Collection (Rockville, MD) and stored at $-80^{\circ} \mathrm{C}$. The virus was propagated in mouse embryo fibroblast (MEF) cell line to prepare for a large virus stock. We infected a total of 20 two-liter roller bottles of MEF cells by MCMV viral stocks at a multiplicity of infection (MOI) of 0.5 . Infected cells were collected when $90 \%$ of cells exhibited cytopathogenic effect (CPE) and pelleted by low speed centrifugation. Cell pellets were then incubated in solution A (1\% NP-40, 20 mmol/L HEPES, $\mathrm{pH} 7.9$, $420 \mathrm{mmol} / \mathrm{L} \mathrm{NaCl}, 1.5 \mathrm{mmol} / \mathrm{L} \mathrm{MgCl}, 0.2 \mathrm{mmol} / \mathrm{L} \mathrm{EDTA}, 0.5 \mathrm{mmo} / \mathrm{L}$ DTT, $25 \%$ glycerol, protease inhibitor mixture) and sonicated mildly with a Branson probe to break cell membranes. Cellular debris was pelleted by low speed centrifugation and removed. Viral particles in the resulting supernatant were pelleted by high speed centrifugation $(55,000 \mathrm{~g})$ for $1 \mathrm{~h}$, resuspended in $15 \mathrm{~mL}$ of solution $\mathrm{A}$, and loaded to a $\mathrm{CsCl}$ density gradient. After $6 \mathrm{~h}$ of high speed centrifugation at $55,000 \mathrm{~g}$, the capsid containing band in the $\mathrm{CsCl}$ gradient was collected, diluted in $5 \mathrm{~mL}$ of buffered saline (PBS), pelleted to remove the residual $\mathrm{CsCl}$ and resuspended in $40 \mathrm{~mL}$ PBS for cryoEM imaging.

\section{CryoEM}

An aliquot of $3 \mathrm{~mL}$ of highly purified MCMV capsid was applied to a
2:1 $\mu \mathrm{m}$ Quantifoil grid coated holey carbon film, blotted by filter paper and plunged into liquid ethane so that MCMV capsids were embedded within vitreous ice suspended across holes of the grids. As detailed below, two sets of cryoEM images were recorded: the first set are CCD images from an FEI Polara G2 cryo electron microscope (i.e., "Polara micrographs") and the second film images from an FEI Titan Krios cryo electron microscope (i.e., "Titan micrographs"), both operated at an accelerating voltage of $300 \mathrm{kV}$ and both with the samples maintained at liquid nitrogen temperature during imaging.

The Polara micrographs were recorded on a TVIPS 16 megapixel CCD camera at a nominal magnification of 59,000* using an electron dose of $\sim 20$ electrons $/ \AA^{2}$. The step size of the CCD image is $1.53 \AA$ pixel at the specimen level. A total of 1796 focal pairs of cryoEM micrographs were recorded, with the first, close-to-focus micrograph aimed at about $-1 \mu \mathrm{m}$ defocus and the second, far-from-focus micrograph at $\sim 3 \mu \mathrm{m}$ defocus. From these Polara micrographs, we obtained $\sim 10,000$ particles for in-depth data processing. However, the best reconstruction we were able to obtain from the Polara micrographs was only at $\sim 11 \AA$ resolution, insufficient for identifying secondary structure elements within the capsid proteins (see below). Therefore, we decided to record higher resolution images in a Titan Krios instrument at $300 \mathrm{kV}$ and obtained 1299 micrographs on Kodak SO163 photographic films (nominal magnification $47,000 \times$ ) with an electron dosage $\sim 20$ electrons $/ \AA^{2}$ and a targeted underfocus value in the range of $1.5-3.0 \mu \mathrm{m}$. The micrographs were digitized with a Nikon Super CoolScan 9000 ED scanner at $6.35 \mu \mathrm{m} / \mathrm{pixel}$, giving a step size of $1.35 \AA /$ pixel at the specimen level.

\section{$3 \mathrm{D}$ reconstruction}

Individual particle images were automatically boxed out from micrographs by the autoBox program in the IMIRS package (Liang et al., 2002), followed by manual screening to select 'good' capsid particle images that appear intact and without contamination and signs of specimen charging. Defocus value and astigmatism parameters of each micrographs were determined with CTFFIND (Mindell and Grigorieff, 2003).

Subsequent data processing includes the determination of particle orientation/center parameters, 3D reconstruction and iterative, projection-based refinement by a distributed computing approach using modular programs in the IMIRS package (Liang et al., 2002) with recent enhancements (Liu et al., 2008). The distributed computing was performed with six Microsoft Windows personal computers and four Windows servers within a custom-designed MPI network. Astigmatism was taken into consideration during the correction of contrast transfer function (CTF) both in the orientation/center refinement step and the $3 \mathrm{D}$ reconstruction step.

Using this procedure, we first obtained a 3D map from 6402 particle images from the Polara micrographs. This map has a resolution of about $11 \AA$ and was used as the starting model to assist processing the higher resolution particle images of the Titan micrographs.

To process the Titan micrographs, we discarded micrographs with specimen charging by evaluating the Fourier transform of the micrographs and selected 669 micrographs for in-depth data processing. From the 669 'good' Titan micrographs selected out through this process, we boxed 5383 particle images and determined their orientation/ center parameters by using the $11 \AA$ map as a starting model. These orientation/center parameters were iteratively refined against the latest 
3D map by gradually including Fourier data at regions of higher spatial frequency. The iterative process was terminated when the reconstruction converges to a stable solution and no further improvement in the resolution of the map was observed. Our reconstruction converges when the high spatial frequency cut-off of the included image data reached $1 / 6.9 \AA^{-1}$. The final map was obtained from 3467 particles images (all from the Titan micrographs) and had a resolution of $9.1 \AA$ based on the 0.5 FSC criterion (Fig. S1).

\section{Segmentation, averaging and visualization of 3D maps}

3D density maps were segmented based on molecular boundaries established by varying displaying threshold (Fig. S6) with UCSF Chimera (Pettersen et al., 2004), in conjunction with other available constraints such as conservation of secondary structural features across nonicosahedral symmetry related copies of the same molecules. Because each hexon contains six subunits, we performed local 6-fold symmetry averaging to improve signal to noise $(\mathrm{S} / \mathrm{N})$ ratio of the hexon map. To do this, a cube of density containing one $C$ hexon was first cut out in Chimera and then rotated by 60 degrees around its local 6 -fold axis. This rotation operation was performed for a total of five times, giving rise to a total of five rotated $C$ hexon maps. The five rotated hexons were then fitted to the original, un-rotated hexon using the 'Fit in Map' function in Chimera to identify the rotation and translation parameters for their best fit. The six hexons were then aligned with the proc3D program of EMAN using the identified parameters of rotation and translation and subsequently averaged with the avg $3 D$ program of EMAN (Ludtke et al., 1999).

To obtain an averaged triplex heterotrimer map, density cubes each containing triplexes Tb, Tc, Td or Te were cut out and aligned using the 'Fit in Map' function in Chimera to identify the rotation and translation parameters for their best fit. The four density cubes were then aligned with the proc $3 D$ program of EMAN using these rotation and translation parameters and subsequently averaged with the avg $3 D$ program of EMAN.

3D visualization and movies were generated with Chimera (Pettersen et al., 2004).

Sequence alignments and structure-constrained homology modeling

We aligned sequence for MCP upper domain (MCPud) of MCMV with sequence of HSV-1 MCPud with the help of Pfam Sequence Search server (Finn et al., 2010) (http://pfam.sanger.ac.uk/search). First we submitted sequence searches to the server one by one and received one Hidden Markov Model (HMM) depicting the consensus sequences among the major capsid proteins of many herpesviruses identified by the Pfam Server. Based on this HMM, we manually aligned the two sequences (Fig. S2).

Secondary structure prediction was carried with online server programs PsiPred (Buchan et al., 2010) and Jpred3 (Cole et al., 2008). To obtain a pseudo-atomic model of the MCMV MCPud using our cryoEM density map as constraints, the primary amino acid sequence of MCMV MCP was submitted to the Phyre ${ }^{2}$ homology model server (http://www.sbg.bio.ic.ac.uk/phyre2) (Kelley and Sternberg, 2009), which first uses a Hidden Markov Model to align the submitted sequence to that of HSV-1 MCP and then generated a homology model using the atomic structure of HSV-1 MCPud (Bowman et al., 2003) as template. The homology model generated by the modeling server was superimposed with the MCMV cryoEM density map of a hexon subunit to assess conformity of secondary structure elements between the two structures with Chimera (Pettersen et al., 2004). Refinement of this preliminary model was performed by adjusting the orientation and position of mismatches of secondary structure elements using Coot (Guex and Peitsch, 1997) to match those revealed in the cryoEM density map. The new model was further refined through several rounds of bond angle and energy optimization via Swiss-PdbViewer (Emsley et al., 2010). This process is iterated several times until a good match between the model and the cryoEM density map was obtained.

\section{ACCESSION NUMBERS}

The cryoEM density map has been deposited to EMDB under the accession number EMD-2435.

\section{ACKNOWLEDGMENTS}

This research was supported by NIH (Al046420). We are grateful to Sanket Shah for assistance in sample purification, to Xiaokang Zhang for assistance in cryoEM imaging during the preliminary phase of the project and to Jonathan Jih for assistance in homology modeling. We thank Xuekui Yu for discussion and Justin Chen for reading our manuscript. We acknowledge the use of the cryoEM facility in the Electron Imaging Center for Nanomachines by NIH (1S10RR23057 to ZHZ) and CNSI at UCLA.

\section{ABBREVIATIONS}

CMV, cytomegalovirus; cryoEM, cryo electron microscopy; HSV-1, herpes simplex virus type 1; MCP, major capsid protein; MCPud, major capsid protein upper domain; SCP, smallest capsid protein; 3D, threedimensional

\section{COMPLIANCE WITH ETHICS GUIDELINES}

Wong H. Hui, Qiyi Tang, Hongrong Liu, Ivo Atanasov, Fenyong Liu, Hua Zhu, Z. Hong Zhou declare that they have no conflict of interest.

This article does not contain any studies with human or animal subjects performed by the any of the authors.

\section{REFERENCES}

Baker, M.L., Jiang, W., Rixon, F.J., and Chiu, W. (2005). Common ancestry of herpesviruses and tailed DNA bacteriophages. J Virol 79, 14967-14970.

Baldick, C.J., Jr., and Shenk, T. (1996). Proteins associated with purified human cytomegalovirus particles. J Virol 70, 6097-6105.

Booy, F.P., Newcomb, W.W., Trus, B.L., Brown, J.C., Baker, T.S., and Steven, A.C. (1991). Liquid-crystalline, phage-like packing of encapsidated DNA in herpes simplex virus. Cell 64, 1007-1015.

Borst, E.M., Mathys, S., Wagner, M., Muranyi, W., and Messerle, M. (2001). Genetic evidence of an essential role for cytomegalovirus small capsid protein in viral growth. J Virol 75, 1450-1458.

Bowman, B.R., Baker, M.L., Rixon, F.J., Chiu, W., and Quiocho, F.A. (2003). Structure of the herpesvirus major capsid protein. EMBO J 22, 757-765.

Britt, W.J., and Boppana, S. (2004). Human cytomegalovirus virion 
proteins. Hum Immunol 65, 395-402.

Buchan, D.W., Ward, S.M., Lobley, A.E., Nugent, T.C., Bryson, K., and Jones, D.T. (2010). Protein annotation and modelling servers at University College London. Nucleic Acids Res 38, W563-568.

Butcher, S.J., Aitken, J., Mitchell, J., Gowen, B., and Dargan, D.J. (1998). Structure of the human cytomegalovirus B capsid by electron cryomicroscopy and image reconstruction. J Struct Biol 124, 70-76.

Chee, M., Rudolph, S.A., Plachter, B., Barrel, B., and Jahn, G. (1989). Identification of the major capsid protein gene of human cytomegalovirus. J Virol 63, 1345-1353.

Chen, D.H., Jiang, H., Lee, M., Liu, F., and Zhou, Z.H. (1999). Threedimensional visualization of tegument/capsid interactions in the intact human cytomegalovirus. Virology 260, 10-16.

Cole, C., Barber, J.D., and Barton, G.J. (2008). The Jpred 3 secondary structure prediction server. Nucleic Acids Res 36, W197-201.

Conway, J.F., Cockrell, S.K., Copeland, A.M., Newcomb, W.W., Brown, J.C., and Homa, F.L. (2010). Labeling and localization of the herpes simplex virus capsid protein UL25 and its interaction with the two triplexes closest to the penton. J Mol Biol 397, 575-586.

Deng, B., O'Connor, C.M., Kedes, D.H., and Zhou, Z.H. (2008). Cryoelectron tomography of Kaposi's sarcoma-associated herpesvirus capsids reveals dynamic scaffolding structures essential to capsid assembly and maturation. J Struct Biol 161, 419-427.

Emsley, P., Lohkamp, B., Scott, W.G., and Cowtan, K. (2010). Features and development of Coot. Acta Crystallogr D Biol Crystallogr 66, 486-501.

Finn, R.D., Mistry, J., Tate, J., Coggill, P., Heger, A., Pollington, J.E., Gavin, O.L., Gunasekaran, P., Ceric, G., Forslund, K., et al. (2010). The Pfam protein families database. Nucleic Acids Res 38, D211-222.

Gibson, W., Baxter, M.K., and Clopper, K.S. (1996a). Cytomegalovirus "missing" capsid protein identified as heat-aggregable product of human cytomegalovirus UL46. J Virol 70, 7454-7461.

Gibson, W., Clopper, K.S., Britt, W.J., and Baxter, M.K. (1996b). Human cytomegalovirus (HCMV) smallest capsid protein identified as product of short open reading frame located between HCMV UL48 and UL49. J Virol 70, 5680-5683.

Guex, N., and Peitsch, M.C. (1997). SWISS-MODEL and the SwissPdbViewer: an environment for comparative protein modeling. Electrophoresis 18, 2714-2723.

Heymann, J.B., Cheng, N., Newcomb, W.W., Trus, B.L., Brown, J.C., and Steven, A.C. (2003). Dynamics of herpes simplex virus capsid maturation visualized by time-lapse cryo-electron microscopy. Nat Struct Biol 10, 334-341.

Homa, F.L., Huffman, J.B., Toropova, K., Lopez, H.R., Makhov, A.M., and Conway, J.F. (2013). Structure of the pseudorabies virus capsid: comparison with herpes simplex virus type 1 and differential binding of essential minor proteins. J Mol Biol. (In Press).

Huang, E., Perkins, E.M., and Desai, P. (2007). Structural features of the scaffold interaction domain at the $\mathrm{N}$ terminus of the major capsid protein (VP5) of herpes simplex virus type 1. J Virol 81, 9396-9407.

Kelley, L.A., and Sternberg, M.J. (2009). Protein structure prediction on the Web: a case study using the Phyre server. Nat Protoc 4, 363-371.

Liang, Y., Ke, E.Y., and Zhou, Z.H. (2002). IMIRS: a high-resolution $3 \mathrm{D}$ reconstruction package integrated with a relational image data- base. J Struct Biol 137, 292-304.

Liu, F., and Zhou, Z.H. (2007). Comparative virion structures of human herpesviruses. In Human Herpesviruses: Biology, Therapy and Immunoprophylaxis. Arvin, A., Campadelli-Fiume, G., Moore, P., Mocarski, E., Roizman, B., Whitley, R., and Yamanishi, K., eds. (Cambridge, UK, Cambridge University Press), pp. 27-43.

Liu, H., Cheng, L., Zeng, S., Cai, C., Zhou, Z.H., and Yang, Q. (2008). Symmetry-adapted spherical harmonics method for high-resolution 3D single-particle reconstructions. J Struct Biol 161, 64-73.

Ludtke, S.J., Baldwin, P.R., and Chiu, W. (1999). EMAN: Semi-automated software for high resolution single particle reconstructions. J Struct Biol 128, 82-97.

Mettenleiter, T.C. (2002). Herpesvirus assembly and egress. J Virol 76, 1537-1547.

Mindell, J.A., and Grigorieff, N. (2003). Accurate determination of local defocus and specimen tilt in electron microscopy. J Struct Biol 142, 334-347.

Mocarski, E.S. (2005). Comparative analysis of herpesvirus-common proteins. In Human Herpesviruses: Biology, Therapy and Immunoprophylaxis. Arvin, A., Campadelli-Fiume, G., Moore, P., Mocarski, E., Roizman, B., Whitley, R., and Yamanishi, K., eds. Cambridge, UK, Cambridge University Press.

Okoye, M.E., Sexton, G.L., Huang, E., McCaffery, J.M., and Desai, P. (2006). Functional analysis of the triplex proteins (VP19C and VP23) of herpes simplex virus type 1. J Virol 80, 929-940.

Pettersen, E.F., Goddard, T.D., Huang, C.C., Couch, G.S., Greenblatt, D.M., Meng, E.C., and Ferrin, T.E. (2004). UCSF Chimera--a visualization system for exploratory research and analysis. J Comput Chem 25, 1605-1612.

Toropova, K., Huffman, J.B., Homa, F.L., and Conway, J.F. (2011). The herpes simplex virus 1 UL17 protein is the second constituent of the capsid vertex-specific component required for DNA packaging and retention. J Virol 85, 7513-7522.

Trus, B.L., Booy, F.P., Newcomb, W.W., Brown, J.C., and Steven, A.C. (1993). Location of VP26 in the herpes simplex virus capsid. Biophys $\mathrm{J} 64, \mathrm{~A} 64$.

Trus, B.L., Gibson, W., Cheng, N., and Steven, A.C. (1999). Capsid structure of simian cytomegalovirus from cryoelectron microscopy: evidence for tegument attachment sites. J Virol 73, 2181-2192.

Trus, B.L., Heymann, J.B., Nealon, K., Cheng, N., Newcomb, W.W., Brown, J.C., Kedes, D.H., and Steven, A.C. (2001). Capsid structure of Kaposi's sarcoma-associated herpesvirus, a gammaherpesvirus, compared to those of an alphaherpesvirus, herpes simplex virus type 1 , and a betaherpesvirus, cytomegalovirus. J Virol 75 , 2879-2890.

Trus, B.L., Newcomb, W.W., Booy, F.P., Brown, J.C., and Steven, A.C. (1992). Distinct monoclonal antibodies separately label the hexons or the pentons of herpes simples virus capsid. Proc Natl Acad Sci USA 89, 11508-11512.

Trus, B.L., Newcomb, W.W., Cheng, N., Cardone, G., Marekov, L., Homa, F.L., Brown, J.C., and Steven, A.C. (2007). Allosteric signaling and a nuclear exit strategy: binding of UL25/UL17 heterodimers to DNA-Filled HSV-1 capsids. Mol Cell 26, 479-489.

Wikoff, W.R., Liljas, L., Duda, R.L., Tsuruta, H., Hendrix, R.W., and Johnson, J.E. (2000). Topologically linked protein rings in the bacteriophage HK97 capsid. Science 289, 2129-2133.

Wu, L., Lo, P., Yu, X., Stoops, J.K., Forghani, B., and Zhou, Z.H. (2000). 
Three-dimensional structure of the human herpesvirus 8 capsid. J Virol 74, 9646-9654.

Yu, X., Shah, S., Atanasov, I., Lo, P., Liu, F., Britt, W.J., and Zhou, Z.H. (2005a). Three-dimensional localization of the smallest capsid protein in the human cytomegalovirus capsid. J Virol 79, 1327-1332.

Yu, X., Trang, P., Shah, S., Atanasov, I., Kim, Y.H., Bai, Y., Zhou, Z.H., and Liu, F. (2005b). Dissecting human cytomegalovirus gene function and capsid maturation by ribozyme targeting and electron cryomicroscopy. Proc Natl Acad Sci U S A 102, 7103-7108.

Yu, X.K., O'Connor, C.M., Atanasov, I., Damania, B., Kedes, D.H., and Zhou, Z.H. (2003). Three-dimensional structures of the A, B, and C capsids of rhesus monkey rhadinovirus: insights into gammaherpesvirus capsid assembly, maturation, and DNA packaging. J Virol 77, 13182-13193.
Zhou, Z.H., Chen, D.H., Jakana, J., Rixon, F.J., and Chiu, W. (1999). Visualization of tegument-capsid interactions and DNA in intact herpes simplex virus type 1 virions. J Virol 73, 3210-3218.

Zhou, Z.H., Dougherty, M., Jakana, J., He, J., Rixon, F.J., and Chiu, W. (2000). Seeing the herpesvirus capsid at $8.5 \AA$. Science 288 , 877-880.

Zhou, Z.H., He, J., Jakana, J., Tatman, J.D., Rixon, F.J., and Chiu, W. (1995). Assembly of VP26 in herpes simplex virus-1 inferred from structures of wild-type and recombinant capsids. Nat Struct Biol 2, 1026-1030.

Zhou, Z.H., Prasad, B.V., Jakana, J., Rixon, F.J., and Chiu, W. (1994). Protein subunit structures in the herpes simplex virus A-capsid determined from $400 \mathrm{kV}$ spot-scan electron cryomicroscopy. J Mol Biol 242, 456-469. 